\title{
Sigmoid colon ureteral fistula presenting with urosepsis
}

\author{
Erich K. Lang, Harry Zinn, Quan D. Nguyen, Raja Anne, Emily Dunn
}

Departments of Urology and Radiology, SUNY, Downstate Health Science Center, NY, USA

This 72-year-old white male presented in the emergency room with symptoms of urosepsis. He had been sick for about ten days, reporting increasing malaise, temperature elevation, left flank pain, and "foul-smelling" urine. He had three prior episodes of left lower quadrant pain associated with diarrhea; which his physician had diagnosed as diverticulitis and treated with antibiotics and dietary restrictions.

At admission, vital signs of the cachectic patient were recorded as BP $160 / 78$, pulse rate 92, respiration 22, and temperature of 38.4 Celsius. Physical exam demonstrated the lungs clear to auscultation and percussion; tenderness to percussion in the left back, and rebound tenderness in the left lower abdominal quadrant. Laboratory data were: RBC 3.8, Hb 9.4, HCT 36, WBC 24,000, BUN 28, Creatinine 2.6; K 4.2, and $\mathrm{Na} \mathrm{Ct} \mathrm{Cl}$ within normal limits. Urinanalysis and cytology demonstrated a murky appearance, specific gravity of 1.024 , cellular debris, WBC 120/hpf, RBC 80/hpf, gram negative bacteria, and vegetable fibers. An admission chest radiograph was negative.

A three-phase contrast-enhanced CT (with intravenous contrast medium reduced to $60 \mathrm{~mL}$, because of elevated creatinine) was performed with both coronal and sagittal reconstructions. An axial slice showed a hugely dilated left ureter with an air fluid level (Figure-1). A coronal reconstruction ( the area of interest enlarged to156\%) demonstrates gas in the fistula to the thickwalled segment of the sigmoid colon as well as at the level of the UPJ (Figure-2). Diverticula are shown in the third portion of the sigmoid colon. An aneurysm of the infra-renal segment of the aorta was noted. Another coronal reconstruction (206\% enlargement of area of interest) at a slightly more posterior level shows the entire left ureter dilated by gas (Figure-3). A sagittal reconstruction shows the dilated gas-filled left ureter (Figure-4).

Fistula from the GI tract to the ureter is uncommon. Uretero-ileal fistulae as consequence of Crohn's disease occur with higher frequency then uretero-colonic fistulae $(1,2)$. Uretero-colonic fistulae may be secondary to inflammatory disease of the large bowel, such as diverticulitis, obstructing ureteral calculi, or neoplasm of the colon that contiguously involves the ureter $(3,4)$. A non-functioning kidney and ureter are often the consequence. CT is the modality of choice in working up urosepsis or renal colic, while antegrade or retrograde ureterogram is the most sensitive in the detection and characterization of a fistulous tract (5). In a like pathologic process, fistulization to the fallopian tubes can occur from diverticular abscesses. However, the incidence of colo-vesical fistulae caused by diverticulitis is higher (6).

Figure 1 - Axial slice showed a hugely dilated left ureter with an air fluid level.

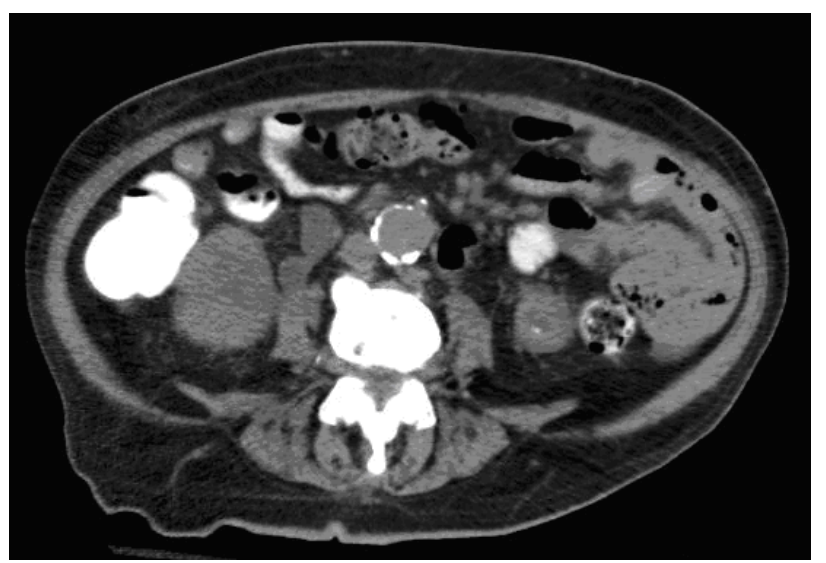


Figure 2 - Axial scan of pelvic CT, (a) noncontrast and (b) contrast enhanced, bone window. A curvilinear hyperdense material (black arrow) was demonstrated at left anterolateral wall of urinary bladder.

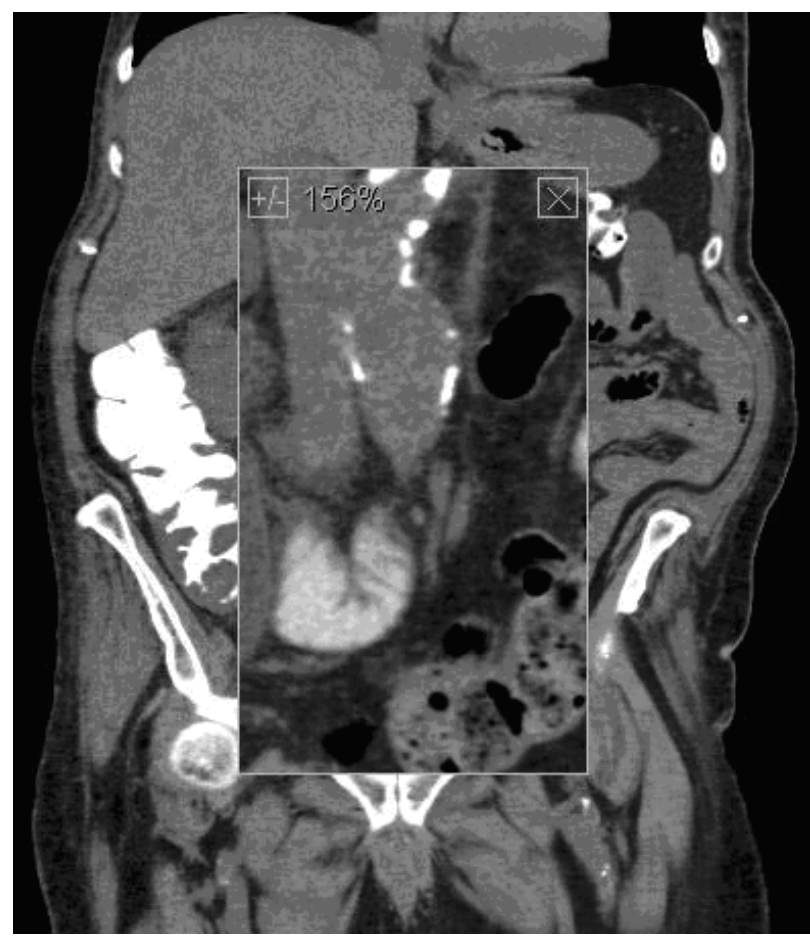

Figure 3 - Coronal reconstruction (206 \% enlargement of area of interest) at a slightly more posterior level shows the entire left ureter dilated by gas.

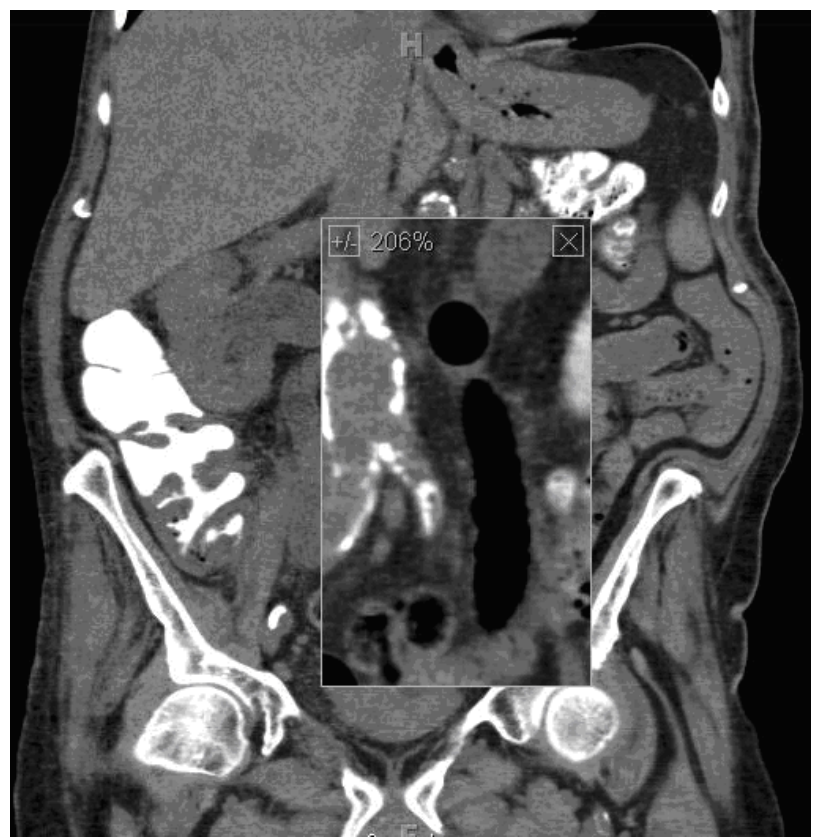

Figure 4 - A sagittal reconstruction shows the dilated gas-filled left ureter.

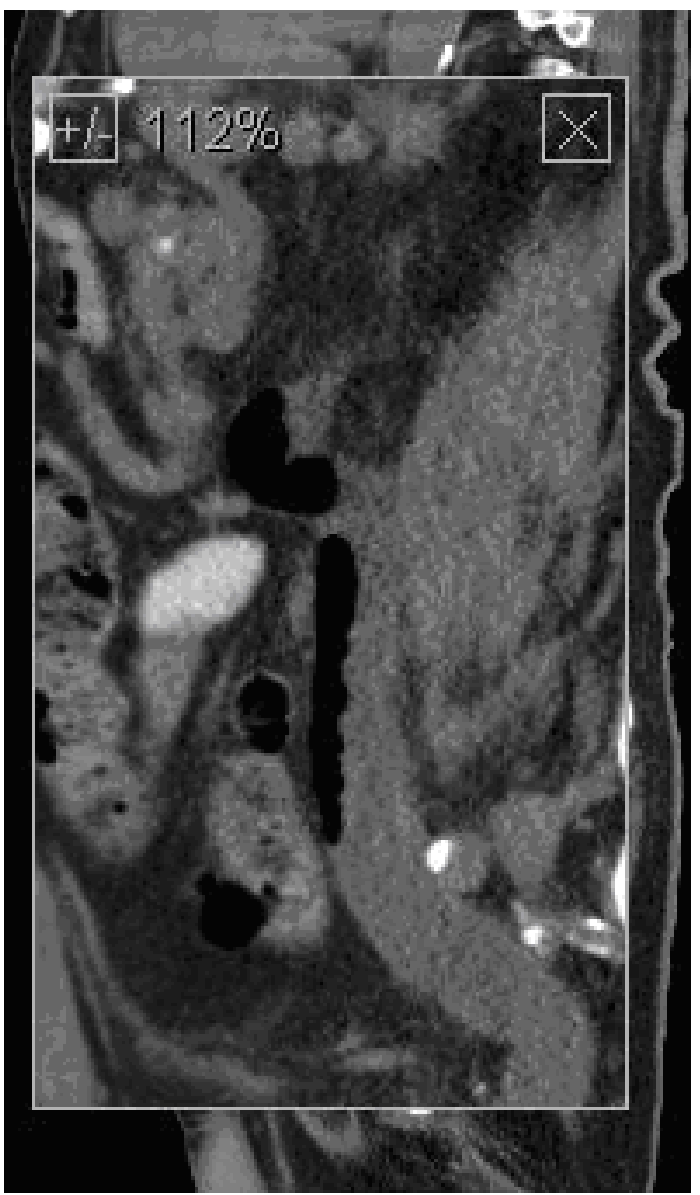

\section{REFERENCES}

1. el Khader K, Karmouni T, Guillé F, Lobel B: Ureteroileal fistula: an unusual complication of Crohn's disease. Acta Gastroenterol Belg. 2000; 63: 312-3.

2. Kleinhans $\mathrm{G}$, Tölle E: Fistula between the bowel and urinary tract in Crohn's disease (author's transl). Urologe A. 1980; 19: 151-5.

3. Conde Santos G, Griñó Garreta J, Bielsa Gali O, Arango Toro 0 , Gelabert-Mas A. Uretero-colonic fistula in non-functioning ureter. Arch Esp Urol. 2001; 54: 1126-9.

4. Cirocco WC, Priolo SR, Golub RW: Spontaneous ureterocolic fistula: a rare complication of colonic diverticular disease. Am Surg. 1994; 60: 832-5. 
5. Lee WK, Chang SD, Roche CJ, Duddalwar VA, Rowley VA, McLoughlin MG: Spontaneous ureterocolic fistula secondary to calculous pyohydroureteronephrosis. $\mathrm{Br} \mathrm{J}$ Radiol. 2005; 78: 954-5.

6. Leicht W, Thomas C, Thüroff J, Roos F: Colovesical fistula caused by diverticulitis of the sigmoid colon: diagnosis and treatment. Urologe A. 2012; 51: 971-4.

Correspondence address:

Dr. Erich K. Lang

Departments of Urology and Radiology SUNY, Downstate Health Science Center

455 Lenox Road

Brooklyn, NY, 11203, USA

E-mail: erich.lang@downstate.edu 